\title{
Weighted Estimates of a Class of Integral Operators with Three Parameters
}

\author{
Ryskul Oinarov ${ }^{1}$ and Aigerim Kalybay ${ }^{2}$ \\ ${ }^{1}$ Eurasian National University, 5 Munaytpasov Street, Astana 010008, Kazakhstan \\ ${ }^{2}$ KIMEP University, 4 Abai Avenue, Almaty 050010, Kazakhstan \\ Correspondence should be addressed to Aigerim Kalybay; kalybay@kimep.kz
}

Received 27 December 2015; Accepted 23 March 2016

Academic Editor: Alberto Fiorenza

Copyright (c) 2016 R. Oinarov and A. Kalybay. This is an open access article distributed under the Creative Commons Attribution License, which permits unrestricted use, distribution, and reproduction in any medium, provided the original work is properly cited.

We characterize the validity of a Hardy-type inequality with a kernel and three parameters $1<p, q, r<\infty$ under some conditions on three weight functions $u, v$, and $w$.

\section{Introduction}

Let $1<p, r<\infty, 0<q<\infty$, and $-\infty \leq a<b \leq+\infty$. Let $u(\cdot), v(\cdot)$, and $w(\cdot)$ be positive functions locally integrable on $(a, b)$, hereinafter referred to as weights. Suppose that for two nonnegative quantities $A$ and $B$ the expression $A \ll B$ means $A \leq C B$ with some constant $C$ that through the paper depends only on the parameters $r, p$, and $q$. The notation $A \approx$ $B$ means $A \ll B \ll A$. Moreover, $1 / p+1 / p^{\prime}=1$.

We consider the following inequalities:

$$
\begin{aligned}
& \left(\int_{a}^{b} u(x)\right. \\
& \left.\cdot\left(\int_{a}^{x}\left(\int_{t}^{x} K(s, t) f(s) d s\right)^{r} w(t) d t\right)^{q / r} d x\right)^{1 / q} \\
& \leq C\left(\int_{a}^{b} v(x) f^{p}(x) d x\right)^{1 / p}, \\
& \left(\int_{a}^{b} u(x)\right. \\
& \left.\cdot\left(\int_{x}^{b}\left(\int_{x}^{t} K(t, s) f(s) d s\right)^{r} w(t) d t\right)^{q / r} d x\right)^{1 / q} \\
& \leq C\left(\int_{a}^{b} v(x) f^{p}(x) d x\right)^{1 / p}
\end{aligned}
$$

for all $f \geq 0$, where the kernel $K(\cdot, \cdot)$ satisfies the conditions

$$
\begin{aligned}
& K(s, t) \geq 0, \\
& a<t \leq s<b, K(s, t) \text { is increasing in } s \text { and decreasing in } t, \\
& K(s, t) \approx K(s, z)+K(z, t)
\end{aligned}
$$

for all $t, z$, and $s$ such that $a<t \leq z \leq s<b$.

A class of Volterra type integral operators with kernels $K(\cdot, \cdot)$ satisfying condition (3) was introduced in [1] and independently in [2]. Later such kernels were considered in many works (see, e.g., [3-8]).

The main aim of this paper is to find necessary and sufficient conditions on the weights $u, v$, and $w$ for the validity of inequalities (1) and (2) in the case $1<p, q, r<\infty$. The same problem for $K(\cdot, \cdot)=1$ was considered in $[9,10]$.

Assume

$$
\begin{aligned}
& A_{0}^{-}(\alpha, \beta)=\sup _{\alpha<x<\beta}\left(\int_{\alpha}^{x} K^{r}(x, s) w(s) d s\right)^{1 / r} \\
& \cdot\left(\int_{x}^{\beta} v^{1-p^{\prime}}(s) d s\right)^{1 / p^{\prime}}, \\
& A_{1}^{-}(\alpha, \beta)=\sup _{\alpha<x<\beta}\left(\int_{\alpha}^{x} w(s) d s\right)^{1 / r}\left(\int_{x}^{\beta} K^{p^{\prime}}(s, x)\right. \\
& \left.\cdot v^{1-p^{\prime}}(s) d s\right)^{1 / p^{\prime}},
\end{aligned}
$$




$$
\begin{aligned}
& A_{0}^{+}(\alpha, \beta)=\sup _{\alpha<x<\beta}\left(\int_{x}^{\beta} K^{r}(s, x) w(s) d s\right)^{1 / r} \\
& \cdot\left(\int_{\alpha}^{x} v^{1-p^{\prime}}(s) d s\right)^{1 / p^{\prime}} \\
& A_{1}^{+}(\alpha, \beta)=\sup _{\alpha<x<\beta}\left(\int_{x}^{\beta} w(s) d s\right)^{1 / r}\left(\int_{\alpha}^{x} K^{p^{\prime}}(x, s)\right. \\
& \left.\cdot v^{1-p^{\prime}}(s) d s\right)^{1 / p^{\prime}} \\
& B_{0}^{-}(\alpha, \beta)=\left(\int_{\alpha}^{\beta}\left(\int_{\alpha}^{x} K^{r}(x, s) w(s) d s\right)^{p /(p-r)}\right. \\
& \left.\cdot\left(\int_{x}^{\beta} v^{1-p^{\prime}}(s) d s\right)^{p(r-1) /(p-r)} v^{1-p^{\prime}}(x) d x\right)^{(p-r) / p r}, \\
& B_{1}^{-}(\alpha, \beta)=\left(\int_{\alpha}^{\beta}\left(\int_{\alpha}^{x} w(s) d s\right)^{r /(p-r)}\right. \\
& \left.\cdot\left(\int_{x}^{\beta} K^{p^{\prime}}(s, x) v^{1-p^{\prime}}(s) d s\right)^{r(p-1) /(p-r)} w(x) d x\right)^{(p-r) / p r}, \\
& B_{0}^{+}(\alpha, \beta)=\left(\int_{\alpha}^{\beta}\left(\int_{x}^{\beta} K^{r}(s, x) w(s) d s\right)^{p /(p-r)}\right. \\
& \left.\cdot\left(\int_{\alpha}^{x} v^{1-p^{\prime}}(s) d s\right)^{p(r-1) /(p-r)} v^{1-p^{\prime}}(x) d x\right)^{(p-r) / p r} \\
& B_{1}^{+}(\alpha, \beta)=\left(\int_{\alpha}^{\beta}\left(\int_{x}^{\beta} w(s) d s\right)^{r /(p-r)}\right. \\
& \left.\cdot\left(\int_{\alpha}^{x} K^{p^{\prime}}(x, s) v^{1-p^{\prime}}(s) d s\right)^{r(p-1) /(p-r)} w(x) d x\right)^{(p-r) / p r}, \\
& U(\alpha, \beta)=\left(\int_{\alpha}^{\beta} u(x) d x\right)^{1 / q} \\
& A^{-}=\max \left\{A_{0}^{-}, A_{1}^{-}\right\}, \\
& A^{+}=\max \left\{A_{0}^{+}, A_{1}^{+}\right\} \text {, } \\
& B^{-}=\max \left\{B_{0}^{-}, B_{1}^{-}\right\}, \\
& B^{+}=\max \left\{B_{0}^{+}, B_{1}^{+}\right\} \text {, } \\
& J^{-}(\alpha, \beta)=\sup _{f \geq 0} \frac{\left(\int_{\alpha}^{\beta}\left(\int_{t}^{\beta} K(s, t) f(s) d s\right)^{r} w(t) d t\right)^{1 / r}}{\left(\int_{\alpha}^{\beta} v(x) f^{p}(x) d x\right)^{1 / p}}, \\
& J^{+}(\alpha, \beta)=\sup _{f \geq 0} \frac{\left(\int_{\alpha}^{\beta}\left(\int_{\alpha}^{t} K(t, s) f(s) d s\right)^{r} w(t) d t\right)^{1 / r}}{\left(\int_{\alpha}^{\beta} v(x) f^{p}(x) d x\right)^{1 / p}} .
\end{aligned}
$$

Two-sided estimates of the values $J^{-}$and $J^{+}$with kernels satisfying condition (3) were found in [11]. Moreover, when $K(\cdot, \cdot)=1$ we get standard Hardy-type estimates that have been extensively investigated by many authors. A complete review of Hardy-type estimates and generalized Hardy-type estimates can be found in books $[12,13]$ and references given there.

The following theorem will be used for the main results.

Theorem A (see [11]). (1) If $1<p \leq r<\infty$, then for all $f \geq 0$ we have

$$
\begin{aligned}
& J^{-} \approx A^{-} \\
& J^{+} \approx A^{+} .
\end{aligned}
$$

(2) If $1<r<p<\infty$, then for all $f \geq 0$ we have

$$
\begin{aligned}
& J^{-} \approx B^{-}, \\
& J^{+} \approx B^{+} .
\end{aligned}
$$

Remark 1. Since the expressions $A_{0}^{ \pm}, A_{1}^{ \pm}, B_{0}^{ \pm}$, and $B_{1}^{ \pm}$are decreasing in $\alpha$ and increasing in $\beta$, then from (5) and (6) we have that $J^{ \pm}(\alpha, \beta)$ are equivalent to a decreasing function in $\alpha$ and an increasing function in $\beta$. This means that there exists a constant $C>0$ depending only on $p$ and $r$ such that $J^{ \pm}(\alpha, \beta) \leq C J^{ \pm}\left(\alpha_{1}, \beta_{1}\right)$ for $\alpha_{1} \leq \alpha<\beta \leq \beta_{1}$.

\section{Main Results}

\subsection{Case $p \leq q$}

Theorem 2. Let $1<p \leq q<\infty$ and $1<r<\infty$. Inequality (1) holds for all $f \geq 0$ if and only if $E^{-}:=$ $\sup _{a<z<b} J^{-}(a, z) U(z, b)<\infty$. Moreover, $E^{-} \approx C$, where $C$ is the best constant in (1).

Theorem 3. Let $1<p \leq q<\infty$ and $1<r<\infty$. Inequality (2) holds for all $f \geq 0$ if and only if $E^{+}:=$ $\sup _{a<z<b} J^{+}(z, b) U(a, z)<\infty$. Moreover, $E^{+} \approx C$, where $C$ is the best constant in (2).

Remark 4. Let us prove only Theorem 2 since the proof of Theorem 3 is similar.

\section{Proof of Theorem 2.}

Sufficiency. Let $E^{-}<\infty$. For any integer $k$ we introduce

$$
\begin{aligned}
x_{k} & =\sup \{a<x \\
& \left.<b: \int_{a}^{x}\left(\int_{t}^{x} K(s, t) f(s) d s\right)^{r} w(t) d t \leq 2^{r k}\right\} .
\end{aligned}
$$


Journal of Function Spaces

3

It is obvious that for any $k$ we have $x_{k} \leq x_{k+1}$. However, when $x_{k}<b$ we have $x_{k}<x_{k+1} \leq b$. Therefore,

$$
\begin{gathered}
\int_{a}^{x_{k}}\left(\int_{t}^{x_{k}} K(s, t) f(s) d s\right)^{r} w(t) d t=2^{r k}, \\
\int_{a}^{x_{k+1}}\left(\int_{t}^{x_{k+1}} K(s, t) f(s) d s\right)^{r} w(t) d t \leq 2^{r(k+1)} .
\end{gathered}
$$

Let $I_{k}=\left[x_{k}, x_{k+1}\right)$. Then

$$
(a, b)=\bigcup_{k}\left[x_{k}, x_{k+1}\right) .
$$

Suppose that $x_{k}<b$; then from (8), twice applying Minkowski's inequality, we get

$$
\begin{aligned}
2^{k-1} & =2^{k}-2^{k-1}=\left(\int_{a}^{x_{k}}\left(\int_{t}^{x_{k}} K(s, t) f(s) d s\right)^{r}\right. \\
\cdot & w(t) d t)^{1 / r} \\
& -\left(\int_{a}^{x_{k-1}}\left(\int_{t}^{x_{k-1}} K(s, t) f(s) d s\right)^{r} w(t) d t\right)^{1 / r} \\
& \leq\left(\int_{x_{k-1}}^{x_{k}}\left(\int_{t}^{x_{k}} K(s, t) f(s) d s\right)^{r} w(t) d t\right)^{1 / r} \\
+ & \left(\int _ { a } ^ { x _ { k - 1 } } \left(\int_{t}^{x_{k-1}} K(s, t) f(s) d s\right.\right. \\
+ & \left.\left.\int_{x_{k-1}}^{x_{k}} K(s, t) f(s) d s\right)^{r} w(t) d t\right)^{1 / r} \\
& -\left(\int_{a}^{x_{k-1}}\left(\int_{t}^{x_{k-1}} K(s, t) f(s) d s\right)^{r} w(t) d t\right)^{1 / r} \\
& +\left(\int_{x_{k-1}}^{x_{k}}\left(\int_{t}^{x_{k}} K(s, t) f(s) d s\right)^{r} w(t) d t\right)^{1 / r} \\
&
\end{aligned}
$$

Since $a<t \leq x_{k-1} \leq s<b$, we can use (3) so that the last gives

$2^{k-1}$

$$
\begin{aligned}
& \ll\left(\int_{x_{k-1}}^{x_{k}}\left(\int_{t}^{x_{k}} K(s, t) f(s) d s\right)^{r} w(t) d t\right)^{1 / r} \\
& \quad+\left(\int_{a}^{x_{k-1}}\left(\int_{x_{k-1}}^{x_{k}} K\left(s, x_{k-1}\right) f(s) d s\right)^{r} w(t) d t\right)^{1 / r} \\
& \quad+\left(\int_{a}^{x_{k-1}}\left(\int_{x_{k-1}}^{x_{k}} K\left(x_{k-1}, t\right) f(s) d s\right)^{r} w(t) d t\right)^{1 / r}
\end{aligned}
$$

$$
\begin{aligned}
= & \left(\int_{x_{k-1}}^{x_{k}}\left(\int_{t}^{x_{k}} K(s, t) f(s) d s\right)^{r} w(t) d t\right)^{1 / r} \\
& +\int_{x_{k-1}}^{x_{k}} K\left(s, x_{k-1}\right) f(s) d s\left(\int_{a}^{x_{k-1}} w(t) d t\right)^{1 / r} \\
& +\int_{x_{k-1}}^{x_{k}} f(s) d s\left(\int_{a}^{x_{k-1}} K^{r}\left(x_{k-1}, t\right) w(t) d t\right)^{1 / r} .
\end{aligned}
$$

From (9) and (10) we have

$$
\begin{aligned}
T & :=\int_{a}^{b} u(x) \\
& \cdot\left(\int_{a}^{x}\left(\int_{t}^{x} K(s, t) f(s) d s\right)^{r} w(t) d t\right)^{q / r} d x \\
& \leq \sum_{k} \int_{x_{k}}^{x_{k+1}} u(x) d x \\
& \cdot\left(\int_{a}^{x_{k+1}}\left(\int_{t}^{x_{k+1}} K(s, t) f(s) d s\right)^{r} w(t) d t\right)^{q / r} \\
& \leq \sum_{k} 2^{q(k+1)} \int_{x_{k}}^{x_{k+1}} u(x) d x \\
& =2^{2 q} \sum_{k} 2^{q(k-1)} \int_{x_{k}}^{x_{k+1}} u(x) d x .
\end{aligned}
$$

From (12) and (13) it follows that

$$
\begin{aligned}
T & \ll 2^{2 q} \sum_{k}\left(\left(\int_{x_{k-1}}^{x_{k}}\left(\int_{t}^{x_{k}} K(s, t) f(s) d s\right)^{r} w(t) d t\right)^{1 / r}\right. \\
& +\int_{x_{k-1}}^{x_{k}} K\left(s, x_{k-1}\right) f(s) d s\left(\int_{a}^{x_{k-1}} w(t) d t\right)^{1 / r} \\
& \left.+\int_{x_{k-1}}^{x_{k}} f(s) d s\left(\int_{a}^{x_{k-1}} K^{r}\left(x_{k-1}, t\right) w(t) d t\right)^{1 / r}\right)^{q} \\
& \cdot \int_{x_{k}}^{x_{k+1}} u(x) d x \\
\ll & \sum_{k}\left(\int_{x_{k-1}}^{x_{k}}\left(\int_{t}^{x_{k}} K(s, t) f(s) d s\right)^{r} w(t) d t\right)^{q / r} \\
& \cdot \int_{x_{k}}^{x_{k+1}} u(x) d x+\sum_{k}\left(\int_{x_{k-1}}^{x_{k}} K\left(s, x_{k-1}\right) f(s) d s\right)^{q} \\
& \cdot\left(\int_{a}^{x_{k-1}} w(t) d t\right)^{q / r} \int_{x_{k}}^{x_{k+1}} u(x) d x \\
& +\sum_{k}\left(\int_{x_{k-1}}^{x_{k}} f(s) d s\right)^{q}\left(\int_{a}^{x_{k-1}} K^{r}\left(x_{k-1}, t\right) w(t) d t\right)^{q / r} \\
& \cdot \int_{x_{k}}^{x_{k+1}} u(x) d x=T_{1}+T_{2}+T_{3} .
\end{aligned}
$$


4

Journal of Function Spaces

Next, we separately estimate $T_{1}, T_{2}$, and $T_{3}$ for $1<p \leq$ $\min \{r, q\}<\infty$ and $1<r<p \leq q<\infty$.

Let $1<p \leq \min \{r, q\}<\infty$. From (5) we get

$$
\begin{aligned}
T_{1} & \ll \sum_{k}\left(J^{-}\left(x_{k-1}, x_{k}\right) U\left(x_{k}, x_{k+1}\right)\right)^{q} \\
& \cdot\left(\int_{x_{k-1}}^{x_{k}} v(t) f^{p}(t) d t\right)^{q / p} \\
& \ll \sum_{k}\left(J^{-}\left(a, x_{k}\right) U\left(x_{k}, b\right)\right)^{q} \\
& \cdot\left(\int_{x_{k-1}}^{x_{k}} v(t) f^{p}(t) d t\right)^{q / p} \leq\left(E^{-}\right)^{q} \\
& \cdot\left(\sum_{k} \int_{x_{k-1}}^{x_{k}} v(t) f^{p}(t) d t\right)^{q / p} \leq\left(E^{-}\right)^{q} \\
& \left(\int_{a}^{b} v(t) f^{p}(t) d t\right)^{q / p} \cdot
\end{aligned}
$$

To estimate $T_{2}$ we use Hölder's inequality:

$$
\begin{aligned}
T_{2} & =\sum_{k}\left(\int_{x_{k-1}}^{x_{k}} K\left(s, x_{k-1}\right) f(s) v^{1 / p}(s) v^{-1 / p}(s) d s\right)^{q} \\
& \cdot\left(\int_{a}^{x_{k-1}} w(t) d t\right)^{q / r} \int_{x_{k}}^{x_{k+1}} u(x) d x, \\
T_{2} & \leq \sum_{k}\left(\int_{a}^{x_{k-1}} w(t) d t\right)^{q / r} \\
& \cdot\left(\int_{x_{k-1}}^{x_{k}} K^{p^{\prime}}\left(s, x_{k-1}\right) v^{1-p^{\prime}}(s) d s\right)^{q / p^{\prime}} \int_{x_{k}}^{x_{k+1}} u(x) d x \\
& \cdot\left(\int_{x_{k-1}}^{x_{k}} v(s) f^{p}(s) d s\right)^{q / p} \\
& \leq \sum_{k}\left(A_{1}^{-}\left(a, x_{k}\right) U\left(x_{k}, b\right)\right)^{q} \\
& =\left(\int_{x_{k-1}}^{x_{k}} v(s) f^{p}(s) d s\right)^{q / p}, \\
T_{2} & \left.\ll \sup _{a<z<b}\left(J^{-}(a, z) U(z, b)\right)^{q}\left(\int_{a}^{b} v(s) f^{p}(s) d s\right)^{q / p}(t) d t\right)^{q / p} \cdot \\
& \\
&
\end{aligned}
$$

To estimate $T_{3}$ we again use Hölder's inequality and get

$$
\begin{aligned}
T_{3} & =\sum_{k}\left(\int_{x_{k-1}}^{x_{k}} f(s) v^{1 / p}(s) v^{-1 / p}(s) d s\right)^{q} \\
& \cdot\left(\int_{a}^{x_{k-1}} K^{r}\left(x_{k-1}, t\right) w(t) d t\right)^{q / r} \int_{x_{k}}^{x_{k+1}} u(x) d x, \\
T_{3} & \leq \sum_{k}\left(\int_{a}^{x_{k-1}} K^{r}\left(x_{k-1}, t\right) w(t) d t\right)^{q / r} \\
& \cdot\left(\int_{x_{k-1}}^{x_{k}} v^{1-p^{\prime}}(s) d s\right)^{q / p^{\prime}} \int_{x_{k}}^{x_{k+1}} u(x) d x \\
& \cdot\left(\int_{x_{k-1}}^{x_{k}} v(s) f^{p}(s) d s\right)^{q / p} \\
& \leq \sum_{k}\left(A_{0}^{-}\left(a, x_{k}\right) U\left(x_{k}, b\right)\right)^{q} \\
& \cdot\left(\int_{x_{k-1}}^{x_{k}} v(s) f^{p}(s) d s\right)^{q / p}, \\
& =\left(E^{-}\right)^{q}\left(\int_{a}^{b} v(t) f^{p}(t) d t\right)^{q / p} \cdot \\
T_{3} & \ll \sup _{a<z<b}\left(J^{-}(a, z) U(z, b)\right)^{q}\left(\int_{a}^{b} v(s) f^{p}(s) d s\right)^{q / p} \\
&
\end{aligned}
$$

From (14), (15), (18), and (21) it follows that for $1<p \leq$ $\min \{r, q\}<\infty$ inequality (1) is correct. Moreover,

$$
C \ll E^{-},
$$

where $C$ is the best constant in (1).

Let us turn to the case $1<r<p \leq q<\infty$. In the same way as above from (6) we get

$$
\begin{aligned}
T_{1} & \ll \sum_{k}\left(J^{-}\left(x_{k-1}, x_{k}\right) U\left(x_{k}, x_{k+1}\right)\right)^{q} \\
& \cdot\left(\int_{x_{k-1}}^{x_{k}} v(t) f^{p}(t) d t\right)^{q / p} \\
& \ll \sum_{k}\left(J^{-}\left(a, x_{k}\right) U\left(x_{k}, b\right)\right)^{q} \\
& \cdot\left(\int_{x_{k-1}}^{x_{k}} v(t) f^{p}(t) d t\right)^{q / p} \leq\left(E^{-}\right)^{q} \\
& \cdot\left(\sum_{k} \int_{x_{k-1}}^{x_{k}} v(t) f^{p}(t) d t\right)^{q / p} \leq\left(E^{-}\right)^{q} \\
& \cdot\left(\int_{a}^{b} v(t) f^{p}(t) d t\right)^{q / p} \cdot
\end{aligned}
$$


To estimate $T_{2}$ we work with (17). Since

$$
\begin{aligned}
& \left(\int_{a}^{x_{k-1}} w(t) d t\right)^{1 / r} \\
& \cdot\left(\int_{x_{k-1}}^{x_{k}} K^{p^{\prime}}\left(s, x_{k-1}\right) v^{1-p^{\prime}}(s) d s\right)^{1 / p^{\prime}} \leq\left(\frac{p-r}{p}\right. \\
& \left.\cdot \int_{a}^{x_{k-1}}\left(\int_{a}^{x} w(t) d t\right)^{r /(p-r)} w(t) d t\right)^{(p-r) / p r} \\
& \quad \cdot\left(\int_{x_{k-1}}^{x_{k}} K^{p^{\prime}}\left(s, x_{k-1}\right) v^{1-p^{\prime}}(s) d s\right)^{1 / p^{\prime}} \ll B_{1}^{-}\left(a, x_{k}\right),
\end{aligned}
$$

we have

$$
\begin{aligned}
T_{2} & \ll \sum_{k}\left(B_{1}^{-}\left(a, x_{k}\right) U\left(x_{k}, b\right)\right)^{q} \\
& \cdot\left(\int_{x_{k-1}}^{x_{k}} v(s) f^{p}(s) d s\right)^{q / p} \\
& \ll \sup _{a<z<b}\left(J^{-}(a, z) U(z, b)\right)^{q}\left(\int_{a}^{b} v(s) f^{p}(s) d s\right)^{q / p} \\
& =\left(E^{-}\right)^{q}\left(\int_{a}^{b} v(t) f^{p}(t) d t\right)^{q / p} .
\end{aligned}
$$

Similarly, working with (20) we have

$$
\begin{gathered}
\left(\int_{a}^{x_{k-1}} K^{r}\left(x_{k-1}, t\right) w(t) d t\right)^{1 / r}\left(\int_{x_{k-1}}^{x_{k}} v^{1-p^{\prime}}(s) d s\right)^{1 / p^{\prime}} \\
\quad \leq\left(\int_{a}^{x_{k-1}} K^{r}\left(x_{k-1}, t\right) w(t) d t\right)^{1 / r}\left(\frac{p-r}{r(p-1)}\right. \\
\cdot \int_{x_{k-1}}^{x_{k}}\left(\int_{x}^{x_{k}} v^{1-p^{\prime}}(s) d s\right)^{p(r-1) /(p-r)} \\
\left.\cdot v^{1-p^{\prime}}(s) d s\right)^{(p-r) / p r} \ll B_{0}^{-}\left(a, x_{k}\right)
\end{gathered}
$$

that yields

$$
\begin{aligned}
T_{3} & \ll \sum_{k}\left(B_{0}^{-}\left(a, x_{k}\right) U\left(x_{k}, b\right)\right)^{q} \\
& \cdot\left(\int_{x_{k-1}}^{x_{k}} v(s) f^{p}(s) d s\right)^{q / p} \\
& \ll \sup _{a<z<b}\left(J^{-}(a, z) U(z, b)\right)^{q}\left(\int_{a}^{b} v(s) f^{p}(s) d s\right)^{q / p} \\
& =\left(E^{-}\right)^{q}\left(\int_{a}^{b} v(t) f^{p}(t) d t\right)^{q / p} .
\end{aligned}
$$

Combining (14), (23), (25), and (27), we have that for $1<$ $r<p \leq q<\infty$ inequality (1) is correct. Moreover,

$$
C \ll E^{-},
$$

where $C$ is the best constant in (1).
Necessity. Let (1) be valid. Let $z \in(a, b)$ and $f:(a, z) \rightarrow$ $R$ be an arbitrary function such that $\int_{a}^{z} v(x) f^{p}(x) d x<\infty$. Suppose that

$$
f_{z}(s)= \begin{cases}f(s), & a<s<z \\ 0, & z \leq s<b\end{cases}
$$

If we substitute the function $f_{z}$ in (1) we have

$$
\begin{aligned}
& \left(\int_{z}^{b} u(x) d x\right)^{1 / q} \\
& \cdot\left(\int_{a}^{z}\left(\int_{t}^{z} K(s, t) f(s) d s\right)^{r} w(t) d t\right)^{1 / r} \\
& \quad \leq C\left(\int_{a}^{z} v(x) f^{p}(x) d x\right)^{1 / p} .
\end{aligned}
$$

From (30) we have

$$
U(z, b) J^{-}(a, z) \leq C \quad \forall z \in(a, b) .
$$

Therefore,

$$
E^{-} \leq C
$$

Moreover, from (22), (28), and (32) we have $C \approx E^{-}$, where $C$ is the best constant in (1). The proof of Theorem 2 is complete.

2.2. Case $q<p$. In this section we consider the case $0<$ $q<p<\infty, p>1$, and $1<r<\infty$ and present sufficient conditions for the validity of inequalities (1) and (2).

Let

$$
\begin{aligned}
F^{-} & =\left(\int_{a}^{b} u(x)\left(\int_{x}^{b} u(s) d s\right)^{q /(p-q)}\right. \\
\cdot & \left.\left(J^{-}(a, x)\right)^{p q /(p-q)} d x\right)^{(p-q) / p q} \\
F^{+} & =\left(\int_{a}^{b} u(x)\left(\int_{a}^{x} u(s) d s\right)^{q /(p-q)}\right. \\
\cdot & \left.\left(J^{+}(x, b)\right)^{p q /(p-q)} d x\right)^{(p-q) / p q} .
\end{aligned}
$$

Theorem 5. Let $0<q<p<\infty, p>1$, and $1<r<\infty$. Inequality (1) holds if $F^{-}<\infty$. Moreover, $C \ll F^{-}$, where $C$ is the best constant in (1).

Theorem 6. Let $0<q<p<\infty, p>1$, and $1<r<\infty$. Inequality (2) holds if $F^{+}<\infty$. Moreover, $C \ll F^{+}$, where $C$ is the best constant in (2).

Remark 7. Let us prove only Theorem 5 since the proof of Theorem 6 is similar. 
6

Journal of Function Spaces

Proof of Theorem 5. The first steps of the proof are similar to those in Theorem 2 up to (14), where

$$
T \ll T_{1}+T_{2}+T_{3} .
$$

This means that we need to separately estimate $T_{1}, T_{2}$, and $T_{3}$.

Let us start with $T_{1}$. Let us notice that we use Hölder's inequality:

$$
\begin{aligned}
T_{1} & =\sum_{k}\left(\int_{x_{k-1}}^{x_{k}}\left(\int_{t}^{x_{k}} K(s, t) f(s) d s\right)^{r} w(t) d t\right)^{q / r} \\
& \cdot \int_{x_{k}}^{x_{k+1}} u(x) d x \leq \sum_{k}\left(J^{-}\left(x_{k-1}, x_{k}\right)\right)^{q} \\
& \cdot \int_{x_{k}}^{x_{k+1}} u(x) d x\left(\int_{x_{k-1}}^{x_{k}} v(t) f^{p}(t) d t\right)^{q / p} \\
& \leq\left(\sum_{k}\left(\int_{x_{k}}^{x_{k+1}} u(x) d x\right)^{p /(p-q)}\right. \\
& \left.\cdot\left(J^{-}\left(x_{k-1}, x_{k}\right)\right)^{p q /(p-q)}\right)^{(p-q) / p}\left(\sum_{k} \int_{x_{k-1}}^{x_{k}} v(t)\right. \\
& \left.\cdot f^{p}(t) d t\right)^{q / p} \ll\left(\sum_{k} \int_{x_{k}}^{x_{k+1}} u(x)\right.
\end{aligned}
$$

$$
\begin{aligned}
& \left(\int_{x}^{x_{k+1}} u(s) d s\right)^{q /(p-q)} d x \\
& \left.\cdot\left(J^{-}\left(x_{k-1}, x_{k}\right)\right)^{p q /(p-q)}\right)^{(p-q) / p}\left(\int_{a}^{b} v(t)\right. \\
& \left.\cdot f^{p}(t) d t\right)^{q / p} \ll\left(\sum_{k} \int_{x_{k}}^{x_{k+1}} u(x)\right. \\
& \left.\cdot\left(\int_{x}^{b} u(s) d s\right)^{q /(p-q)}\right)^{(p-q) / p}\left(\int_{a}^{b} v(t)\right. \\
& \left.\left.\cdot\left(J^{-}(a, x)\right)^{p q /(p-q)} d x\right)^{q}(t) d t\right)^{q / p} \leq\left(F^{-}\right)^{q}\left(\int_{a}^{b} v(t)\right. \\
& \left.\cdot f^{p}(t) d t\right)^{q / p} \cdot
\end{aligned}
$$

Now, we turn to the estimation of $T_{2}$. Again Hölder's inequality is used:

$$
\begin{aligned}
& T_{2}=\sum_{k}\left(\int_{x_{k-1}}^{x_{k}} K\left(s, x_{k-1}\right) f(s) d s\right)^{q}\left(\int_{a}^{x_{k-1}} w(t) d t\right)^{q / r} \int_{x_{k}}^{x_{k+1}} u(x) d x \leq \sum_{k}\left(\int_{x_{k-1}}^{x_{k}} K^{p^{\prime}}\left(s, x_{k-1}\right) v^{1-p^{\prime}}(s) d s\right)^{q / p^{\prime}} \\
& \cdot\left(\int_{a}^{x_{k-1}} w(t) d t\right)^{q / r} \int_{x_{k}}^{x_{k+1}} u(x) d x\left(\int_{x_{k-1}}^{x_{k}} v(t) f^{p}(t) d t\right)^{q / p} \leq\left(\sum_{k}\left(\int_{x_{k}}^{x_{k+1}} u(x) d x\right)^{p /(p-q)}\right. \\
& \left.\cdot\left(\int_{x_{k-1}}^{x_{k}} K^{p^{\prime}}\left(s, x_{k-1}\right) v^{1-p^{\prime}}(s) d s\right)^{q(p-1) /(p-q)}\left(\int_{a}^{x_{k-1}} w(t) d t\right)^{q p / r(p-q)}\right)^{(p-q) / p}\left(\sum_{k} \int_{x_{k-1}}^{x_{k}} v(t) f^{p}(t) d t\right)^{q / p} \\
& \leq\left(\sum_{k}\left(\int_{x_{k}}^{x_{k+1}} u(x) d x\right)^{p /(p-q)}\right. \\
& \left.\cdot\left[\left(\int_{a}^{x_{k-1}} w(t) d t\right)^{p /(p-r)}\left(\int_{x_{k-1}}^{x_{k}} K^{p^{\prime}}\left(s, x_{k-1}\right) v^{1-p^{\prime}}(s) d s\right)^{r(p-1) /(p-r)}\right]^{q(p-r) / r(p-q)}\right)^{(p-q) / p}\left(\int_{a}^{b} v(t) f^{p}(t) d t\right)^{q / p} \\
& \ll\left(\sum_{k} \int_{x_{k}}^{x_{k+1}} u(x)\left(\int_{x}^{x_{k+1}} u(s) d s\right)^{q /(p-q)} d x\right. \\
& \left.\cdot\left[\int_{a}^{x_{k-1}} w(t)\left(\int_{a}^{t} w(\tau) d \tau\right)^{r /(p-r)} d t\left(\int_{x_{k-1}}^{x_{k}} K^{p^{\prime}}\left(s, x_{k-1}\right) v^{1-p^{\prime}}(s) d s\right)^{r(p-1) /(p-r)}\right]^{q(p-r) / r(p-q)}\right)^{(p-q) / p} \\
& \cdot\left(\int_{a}^{b} v(t) f^{p}(t) d t\right)^{q / p} \leq\left(\sum_{k} \int_{x_{k}}^{x_{k+1}} u(x)\left(\int_{x}^{b} u(s) d s\right)^{q /(p-q)} d x\right.
\end{aligned}
$$


Journal of Function Spaces

7

$$
\begin{aligned}
& \left.\cdot\left(\left[\int_{a}^{x_{k-1}} w(t)\left(\int_{a}^{t} w(\tau) d \tau\right)^{r /(p-r)}\left(\int_{t}^{x_{k}} K^{p^{\prime}}(s, t) v^{1-p^{\prime}}(s) d s\right)^{r(p-1) /(p-r)} d t\right]^{(p-r) / p r}\right)^{p q /(p-q)}\right)^{(p-q) / p} \\
& \cdot\left(\int_{a}^{b} v(t) f^{p}(t) d t\right)^{q / p} \leq\left(\int_{a}^{b} u(x)\left(\int_{x}^{b} u(s) d s\right)^{q /(p-q)}\left(B_{1}^{-}(a, x)\right)^{p q /(p-q)} d x\right)^{(p-q) / p}\left(\int_{a}^{b} v(t) f^{p}(t) d t\right)^{q / p} \\
& \ll\left(\int_{a}^{b} u(x)\left(\int_{x}^{b} u(s) d s\right)^{q /(p-q)}\left(J^{-}(a, x)\right)^{p q /(p-q)} d x\right)^{(p-q) / p}\left(\int_{a}^{b} v(t) f^{p}(t) d t\right)^{q / p}=\left(F^{-}\right)^{q} \\
& \cdot\left(\int_{a}^{b} v(t) f^{p}(t) d t\right)^{q / p} \cdot
\end{aligned}
$$

The last step is to estimate $T_{3}$ :

$$
\begin{aligned}
& T_{3}=\sum_{k}\left(\int_{x_{k-1}}^{x_{k}} f(s) d s\right)^{q}\left(\int_{a}^{x_{k-1}} K^{r}\left(x_{k-1}, t\right) w(t) d t\right)^{q / r} \int_{x_{k}}^{x_{k+1}} u(x) d x \leq \sum_{k}\left(\int_{x_{k-1}}^{x_{k}} v^{1-p^{\prime}}(s) d s\right)^{q / p^{\prime}}\left(\int_{a}^{x_{k-1}} K^{r}\left(x_{k-1}, t\right)\right. \\
& \cdot w(t) d t)^{q / r} \int_{x_{k}}^{x_{k+1}} u(x) d x\left(\int_{x_{k-1}}^{x_{k}} v(s) f^{p}(s) d s\right)^{q / p} \leq\left(\sum_{k}\left(\int_{x_{k}}^{x_{k+1}} u(x) d x\right)^{p /(p-q)}\right. \\
& \left.\cdot\left[\left(\int_{a}^{x_{k-1}} K^{r}\left(x_{k-1}, t\right) w(t) d t\right)^{q / r}\left(\int_{x_{k-1}}^{x_{k}} v^{1-p^{\prime}}(s) d s\right)^{q / p^{\prime}}\right]^{p /(p-q)}\right)^{(p-q) / p}\left(\int_{a}^{b} v(s) f^{p}(s) d s\right)^{q / p} \\
& \leq\left(\sum _ { k } ( \int _ { x _ { k } } ^ { x _ { k + 1 } } u ( x ) d x ) ^ { p / ( p - q ) } \left(\left[\left(\int_{x_{k-1}}^{x_{k}} v^{1-p^{\prime}}(s) d s\right)^{r(p-1) /(p-r)}\right.\right.\right. \\
& \left.\left.\left.\cdot\left(\int_{a}^{x_{k-1}} K^{r}\left(x_{k-1}, t\right) w(t) d t\right)^{p /(p-r)}\right]^{(p-r) / p r}\right)^{p q /(p-q)}\right)^{(p-q) / p}\left(\int_{a}^{b} v(s) f^{p}(s) d s\right)^{q / p} \ll\left(\sum_{k} \int_{x_{k}}^{x_{k+1}} u(x)\right. \\
& \cdot\left(\int_{x}^{b} u(\tau) d \tau\right)^{q /(p-q)} d x\left(\left[\int_{x_{k-1}}^{x_{k}} v^{1-p^{\prime}}(s)\left(\int_{s}^{x_{k}} v^{1-p^{\prime}}(t) d t\right)^{p(r-1) /(p-r)} d s\right.\right. \\
& \left.\left.\left.\cdot\left(\int_{a}^{x_{k-1}} K^{r}\left(x_{k-1}, t\right) w(t) d t\right)^{p /(p-r)}\right]^{(p-r) / p r}\right)^{p q /(p-q)}\right)^{(p-q) / p}\left(\int_{a}^{b} v(s) f^{p}(s) d s\right)^{q / p} \leq\left(\sum_{k} \int_{x_{k}}^{x_{k+1}} u(x)\right. \\
& \cdot\left(\int_{x}^{b} u(\tau) d \tau\right)^{q /(p-q)} \\
& \left.\cdot\left(\left[\int_{a}^{x} v^{1-p^{\prime}}(s)\left(\int_{s}^{x} v^{1-p^{\prime}}(t) d t\right)^{p(r-1) /(p-r)}\left(\int_{a}^{s} K^{r}(s, t) w(t) d t\right)^{p /(p-r)} d s\right]^{(p-r) / p r}\right)^{p q /(p-q)} d x\right)^{(p-q) / p} \\
& \cdot\left(\int_{a}^{b} v(s) f^{p}(s) d s\right)^{q / p} \leq\left(\int_{a}^{b} u(x)\left(\int_{x}^{b} u(\tau) d \tau\right)^{q /(p-q)}\left(B_{0}^{-}(a, x)\right)^{p q /(p-q)} d x\right)^{(p-q) / p}\left(\int_{a}^{b} v(s) f^{p}(s) d s\right)^{q / p} \\
& \ll\left(\int_{a}^{b} u(x)\left(\int_{x}^{b} u(\tau) d \tau\right)^{q /(p-q)}\left(J^{-}(a, x)\right)^{p q /(p-q)} d x\right)^{(p-q) / p}\left(\int_{a}^{b} v(s) f^{p}(s) d s\right)^{q / p}=\left(F^{-}\right)^{q}\left(\int_{a}^{b} v(s)\right. \\
& \left.\cdot f^{p}(s) d s\right)^{q / p} \text {. }
\end{aligned}
$$


Combining (14), (35), (36), and (37), we have that inequality (1) is correct. Moreover, $C \ll F^{-}$, where $C$ is the best constant in (1).

Remark 8. Let us consider the following inequalities:

$$
\begin{aligned}
& \left(\int_{a}^{b} u(x)\right. \\
& \left.\cdot\left(\int_{a}^{x}\left(\int_{t}^{b} K(s, t) f(s) d s\right)^{r} w(t) d t\right)^{q / r} d x\right)^{1 / q} \\
& \leq \widetilde{C}\left(\int_{a}^{b} v(x) f^{p}(x) d x\right)^{1 / p}, \\
& \left(\int_{a}^{b} u(x)\right. \\
& \left.\cdot\left(\int_{x}^{b}\left(\int_{a}^{t} K(t, s) f(s) d s\right)^{r} w(t) d t\right)^{q / r} d x\right)^{1 / q} \\
& \quad \leq \widetilde{C}\left(\int_{a}^{b} v(x) f^{p}(x) d x\right)^{1 / p} \cdot
\end{aligned}
$$

It is obvious that, in view of (3), the validity of inequality (38) is equivalent to the simultaneous validity of inequality (1) and the following inequalities:

$$
\begin{aligned}
& \left(\int_{a}^{b} u(x)\left(\int_{a}^{x} w(t) d t\right)^{q / r}\right. \\
& \left.\cdot\left(\int_{x}^{b} K(s, x) f(s) d s\right)^{q} d x\right)^{1 / q} \leq \widetilde{C_{1}}\left(\int_{a}^{b} v(t)\right. \\
& \left.\cdot f^{p}(t) d t\right)^{1 / p}, \\
& \left(\int_{a}^{b} u(x)\left(\int_{a}^{x} K^{r}(x, t) w(t) d t\right)^{q / r}\right. \\
& \left.\cdot\left(\int_{x}^{b} f(s) d s\right)^{q} d x\right)^{1 / q} \leq \widetilde{C_{2}}\left(\int_{a}^{b} v(t)\right. \\
& \left.\cdot f^{p}(t) d t\right)^{1 / p} \cdot
\end{aligned}
$$

Inequality (40) can be treated by Theorem A, while inequality (41) is the standard Hardy-type inequality. This means that if we combine Theorem 2 and the known results on Hardytype inequalities, we can characterize (38) for the case $1<$ $p \leq q<\infty$ and $1<r<\infty$. Similar splitting can be done for inequality (39). In [14] inequalities (38) and (39) are completely characterized for all relations between $p, q$, and $r$, where $1 \leq p<\infty, 0<q<\infty$, and $0<r \leq \infty$. The characterization method in [14] is not based on the integral splitting. Thus, due to the splitting, our main inequalities (1) and (2) allow characterizing inequalities (38) and (39). However, inversely, inequalities (38) and (39) do not help to characterize inequalities (1) and (2).

Let us also notice that when $K(\cdot, \cdot)=1$ inequalities (38) and (39) were considered in [15-17].

\section{Applications}

(1) Let a function $g: I \rightarrow R$ have generalized derivatives up to $n$th order; $n>1$. Let $0 \leq k \leq n-1$. Now we consider the inequality

$$
\|\| R_{n-k}\left(\cdot, \cdot, g^{(k)}\right)\left\|_{r, w}\right\|_{q, u} \leq C\left\|g^{(n)}\right\|_{p, v}
$$

where the inside norm $\left\|R_{n-k}\left(\cdot, \cdot, g^{(k)}\right)\right\|_{r, w}$ is taken with respect to the second argument of the function $R_{n-k}$ and the function $R_{n-k}\left(t, x, g^{(k)}\right)$ is the $(n-k)$ th remainder of Taylor's formula of $g^{(k)}$; that is,

$$
R_{n-k}\left(t, x, g^{(k)}\right)=g^{(k)}(t)-\sum_{i=0}^{n-k-1} \frac{g^{(k+i)}(x)(t-x)^{i}}{i !}
$$

In the case $k=0$ we have

$$
R_{n}(t, x, g)=g(t)-\sum_{i=0}^{n-1} \frac{g^{(i)}(x)(t-x)^{i}}{i !}
$$

Moreover, $\|\cdot\|_{p, v}$ stands for

$$
\|f\|_{p, v}=\left(\int_{a}^{b} v(x)|f(x)|^{p} d x\right)^{1 / p}
$$

By integration by parts it is easy to see that for $x>t$ we have

$$
\begin{aligned}
& R_{n-k}\left(t, x, g^{(k)}\right) \\
& \quad=\frac{(-1)^{n-k}}{(n-k-1) !} \int_{t}^{x}(s-t)^{n-k-1} g^{(n)}(s) d s \\
& \quad:=G_{k}^{-}\left(t, x, g^{(n)}\right) .
\end{aligned}
$$

Similarly, for $x<t$ we get

$$
\begin{aligned}
& R_{n-k}\left(t, x, g^{(k)}\right) \\
& \quad=\frac{1}{(n-k-1) !} \int_{x}^{t}(t-s)^{n-k-1} g^{(n)}(s) d s \\
& \quad:=G_{k}^{+}\left(t, x, g^{(n)}\right) .
\end{aligned}
$$


Journal of Function Spaces

9

Therefore, inequality (42) holds if and only if the following inequalities simultaneously hold:

$$
\begin{aligned}
& \left(\int_{a}^{b} u(x)\left(\int_{a}^{x}\left|G_{k}^{-}\left(t, x, g^{(n)}\right)\right|^{r} w(t) d t\right)^{q / r} d x\right)^{1 / q} \\
& \leq C\left(\int_{a}^{b} v(x)\left|g^{(n)}(x)\right|^{p} d x\right)^{1 / p}, \\
& \left(\int_{a}^{b} u(x)\left(\int_{x}^{b}\left|G_{k}^{+}\left(t, x, g^{(n)}\right)\right|^{r} w(t) d t\right)^{q / r} d x\right)^{1 / q} \\
& \leq C\left(\int_{a}^{b} v(x)\left|g^{(n)}(x)\right|^{p} d x\right)^{1 / p} .
\end{aligned}
$$

Thus, if we denote $E_{k}^{ \pm}:=E^{ \pm}$and $F_{k}^{ \pm}:=F^{ \pm}$when $K(s, t)=$ $(s-t)^{n-k-1}$, from Theorems $2,3,5$, and 6 we have the following.

Theorem 9. Let $0 \leq k \leq n-1$. Let $1<p \leq q<\infty$ and $1<r<$ $\infty$. Inequality (42) holds if and only if $E_{k}=\max \left\{E_{k}^{-}, E_{k}^{+}\right\}<\infty$. Moreover, $E_{k} \approx C$, where $C$ is the best constant in (42).

Theorem 10. Let $0 \leq k \leq n-1$. Let $0<q<p<\infty, p>1$, and $1<r<\infty$. Inequality (42) holds if $F_{k}=\max \left\{F_{k}^{-}, F_{k}^{+}\right\}<\infty$. Moreover, $C \ll F_{k}$, where $C$ is the best constant in (42).

Remark 11. If inequality (42) holds, then the inequalities

$$
\begin{aligned}
& \left(\int_{a}^{b} u(x)\left(\int_{a}^{b}\left|g^{(n-1)}(x)-g^{(n-1)}(t)\right|^{r} w(t) d t\right)^{q / r} d x\right)^{1 / q} \leq C\left(\int_{a}^{b} v(x)\left|g^{(n)}(x)\right|^{p} d x\right)^{1 / p} \\
& \left(\int_{a}^{b} u(x)\left(\int_{a}^{b}\left|g^{(k)}(x)-g^{(k)}(t)\right|^{r} w(t) d t\right)^{q / r} d x\right)^{1 / q} \\
& \leq C\left(\left(\int_{a}^{b} v(x)\left|g^{(n)}(x)\right|^{p} d x\right)^{1 / p}+\sum_{i=k+1}^{n-1}\left(\int_{a}^{b} u(x)\left(\int_{a}^{b}\left|g^{(i)}(t)(x-t)^{i-k}\right|^{r} w(t) d t\right)^{q / r} d x\right)^{1 / q}\right), 0 \leq k<n-1,
\end{aligned}
$$

also hold.

(2) In this part of the paper we investigate the inequality

$$
\begin{aligned}
& \left(\int_{a}^{b} u(x)\left(\int_{a}^{b}\left|g^{(k)}(x)-g^{(k)}(t)\right|^{r} w(t) d t\right)^{q / r} d x\right)^{1 / q} \\
& \quad \leq C\left(\int_{a}^{b} v(x)\left|g^{(n)}(x)\right|^{p} d x\right)^{1 / p}
\end{aligned}
$$

for $0 \leq k<n-1$ with the conditions

$$
\begin{aligned}
\lim _{t \rightarrow a^{+}} g^{(i)}(t)=g^{(i)}(a)= & \\
\lim _{t \rightarrow b^{-}} g^{(i)}(t)=g^{(i)}(b)= & \\
& \text { for } i=k, k+1, \ldots, n-1 .
\end{aligned}
$$

When $r=q$ inequality (50) turns to the inequality

$$
\begin{aligned}
& \left(\int_{a}^{b} \int_{a}^{b}\left|g^{(k)}(x)-g^{(k)}(t)\right|^{q} u(x) w(t) d t d x\right)^{1 / q} \\
& \quad \leq C\left(\int_{a}^{b} v(x)\left|g^{(n)}(x)\right|^{p} d x\right)^{1 / p} .
\end{aligned}
$$

Characterization of inequality (52) with conditions (51) is associated with open problem 2 in book [13, page 297].
First, we consider the inequality

$$
\begin{aligned}
& \left(\int_{a}^{b} u(x)\left(\int_{a}^{x}\left|g^{(k)}(x)-g^{(k)}(t)\right|^{r} w(t) d t\right)^{q / r} d x\right)^{1 / q} \\
& \quad \leq C\left(\int_{a}^{b} v(x)\left|g^{(n)}(x)\right|^{p} d x\right)^{1 / p}
\end{aligned}
$$

for $0 \leq k<n-1$ with the conditions

$$
\lim _{t \rightarrow b^{-}} g^{(i)}(t)=g^{(i)}(b)=0 \quad \text { for } i=k, k+1, \ldots, n-1
$$

Let $g^{(n)}(t)=f(t)$; then we have

$$
\begin{array}{r}
g^{(k)}(t)=\frac{(-1)^{n-k}}{(n-k-1) !} \int_{t}^{b}(s-t)^{n-k-1} f(s) d s, \\
\quad k=0,1, \ldots, n-1 .
\end{array}
$$

Therefore, for $x>t$,

$$
\begin{aligned}
g^{(k)}(t)-g^{(k)}(x) & \\
= & \frac{(-1)^{n-k}}{(n-k-1) !}\left[\int_{t}^{b}(s-x)^{n-k-1} f(s) d s\right. \\
& \left.-\int_{x}^{b}(s-t)^{n-k-1} f(s) d s\right]
\end{aligned}
$$


10

Journal of Function Spaces

$$
\begin{aligned}
& =\frac{(-1)^{n-k}}{(n-k-1) !}\left[\int_{t}^{x}(s-t)^{n-k-1} f(s) d s\right. \\
& \left.+\int_{x}^{b}\left[(s-t)^{n-k-1}-(s-x)^{n-k-1}\right] f(s) d s\right] .
\end{aligned}
$$

In the case $n>2$ and $k=n-2$ we have

$$
\begin{aligned}
g^{(n-2)}(t)-g^{(n-2)}(x)= & \int_{t}^{x}(s-t) f(s) d s \\
& +(x-t) \int_{x}^{b} f(s) d s .
\end{aligned}
$$

In the case $n>2$ and $0 \leq k<n-2$ for $s \geq x \geq t$ we use the following relation:

$$
\begin{aligned}
& (s-t)^{n-k-1}-(s-x)^{n-k-1} \\
& \quad \approx(x-t)\left[(s-x)^{n-k-2}+(x-t)^{n-k-2}\right] .
\end{aligned}
$$

From (56), (57), and (58) we have

$$
\begin{aligned}
& g^{(k)}(x)-g^{(k)}(t) \\
& \approx \int_{t}^{x}(s-t)^{n-k-1} f(s) d s+(x-t)^{n-k-1} \int_{x}^{b} f(s) d s \\
& \quad+\gamma(k, n)(x-t) \int_{x}^{b}(s-t)^{n-k-2} f(s) d s
\end{aligned}
$$

where $\gamma(k, n)=0$ when $k=n-2$ and $\gamma(k, n)=1$ when $0 \leq k<n-2$.

From (59) we obtain

$$
\begin{aligned}
& \left(\int_{a}^{x}\left|g^{(k)}(x)-g^{(k)}(t)\right|^{r} w(t) d t\right)^{1 / r} \\
& \quad \approx\left(\int_{a}^{x}\left|\int_{t}^{x}(s-t)^{n-k-1} f(s) d s\right|^{r} w(t) d t\right)^{1 / r} \\
& +\left(\int_{a}^{x}(x-t)^{r(n-k-1)} w(t) d t\right)^{1 / r}\left|\int_{x}^{b} f(s) d s\right| \\
& +\gamma(k, n)\left(\int_{a}^{x}(x-t)^{r} w(t) d t\right)^{1 / r} \\
& \quad \cdot\left|\int_{x}^{b}(s-t)^{n-k-2} f(s) d s\right| .
\end{aligned}
$$

Consequently, the validity of inequality (53) is equivalent to the simultaneous validity of the following inequalities:

$$
\begin{aligned}
& \left(\int_{a}^{b} u(x)\right. \\
& \left.\cdot\left(\int_{a}^{x}\left(\int_{t}^{x}(s-t)^{n-k-1} f(s) d s\right)^{r} w(t) d t\right)^{q / r} d x\right)^{1 / q} \\
& \leq C_{1}\left(\int_{a}^{b} v(x) f^{p}(x) d x\right)^{1 / p}, \\
& \left(\int_{a}^{b} W_{m}^{-}(x)\left(\int_{x}^{b}(s-x)^{m-k} f(s) d s\right)^{q} d x\right)^{1 / q} \\
& \leq C_{2}\left(\int_{a}^{b} v(x) f^{p}(x) d x\right)^{1 / p}
\end{aligned}
$$

for $m=k$ and $m=n-2$, where $W_{k}^{-}(x)=u(x)\left(\int_{a}^{x}(x-\right.$ $\left.t)^{r(n-k-1)} w(t) d t\right)^{1 / r}, 0 \leq k<n-1$.

Inequality (61) can be characterized by Theorems 2 and 5 when $K(s, t)=(s-t)^{n-k-1}$.

Inequality (62) can be characterized by Theorem $A$ if we denote $A_{q, m}^{-}(a, b):=A^{-}(a, b)$ and $B_{q, m}^{-}(a, b):=B^{-}(a, b)$, where we replace $K(s, t)$ by $(s-t)^{m-k}, w$ by $W_{m}^{-}$, and $r$ by $q$. Then by Theorem A inequality (62) is valid if and only if $A_{q, m}^{-}(a, b)<\infty$ for $1<p \leq q<\infty$ and $B_{q, m}^{-}(a, b)<\infty$ for $1<q<p<\infty$.

Thus, the following hold.

Proposition 12. Let $n \geq 2$ and $0 \leq k<n-1$. Let $1<p \leq q<\infty$ and $1<r<\infty$. Suppose that $g$ satisfies condition (54). Then inequality (53) holds if and only if $D_{k}^{-}=\max \left\{\gamma(k, n) A_{q, n-2}^{-}(a, b), A_{q, k}^{-}(a, b), E_{k}^{-}\right\}<\infty$. Moreover, $D_{k}^{-} \approx C$, where $C$ is the best constant in (53).

Proposition 13. Let $n \geq 2$ and $0 \leq k<n-1$. Let $1<q<p<\infty$ and $1<r<\infty$. Suppose that $g$ satisfies condition (54). Then inequality (53) holds if $M_{k}^{-}=$ $\max \left\{\gamma(k, n) B_{q, n-2}^{-}(a, b), B_{q, k}^{-}(a, b), F_{k}^{-}\right\}<\infty$. Moreover, $M_{k}^{-} \approx$ $C$, where $C$ is the best constant in (53).

A similar result can be written for the inequality

$$
\begin{aligned}
& \left(\int_{a}^{b} u(x)\left(\int_{x}^{b}\left|g^{(k)}(t)-g^{(k)}(x)\right|^{r} w(t) d t\right)^{q / r} d x\right)^{1 / q} \\
& \leq C\left(\int_{a}^{b} v(x)\left|g^{(n)}(x)\right|^{p} d x\right)^{1 / p}
\end{aligned}
$$

with the conditions

$$
\lim _{t \rightarrow a^{+}} g^{(i)}(t)=g^{(i)}(a)=0 \quad \text { for } i=k, k+1, \ldots, n-1 .
$$

Here we need the following notations: $A_{q, m}^{+}(a, b):=A^{+}(a, b)$ and $B_{q, m}^{+}(a, b):=B^{+}(a, b)$, where we replace $K(s, t)$ by 
$(s-t)^{m-k}, w$ by $W_{m}^{+}$for $m=k$ and $m=n-2$, where $W_{k}^{+}(x)=u(x)\left(\int_{x}^{b}(t-x)^{r(n-k-1)} w(t) d t\right)^{1 / r}, 0 \leq k<n-1$, and $r$ by $q$.

Proposition 14. Let $n \geq 2$ and $0 \leq k<n-1$. Let $1<p \leq q<\infty$ and $1<r<\infty$. Suppose that $g$ satisfies condition (64). Then inequality (63) holds if and only if $D_{k}^{+}=\max \left\{\gamma(k, n) A_{q, n-2}^{+}(a, b), A_{q, k}^{+}(a, b), E_{k}^{+}\right\}<\infty$. Moreover, $D_{k}^{+} \approx C$, where $C$ is the best constant in (63).

Proposition 15. Let $n \geq 2$ and $0 \leq k<n-1$. Let $1<q<p<\infty$ and $1<r<\infty$. Suppose that $g$ satisfies condition (64). Then inequality (63) holds if $M_{k}^{+}=$ $\max \left\{\gamma(k, n) B_{q, n-2}^{+}(a, b), B_{q, k}^{+}(a, b), F_{k}^{+}\right\}<\infty$. Moreover, $M_{k}^{+} \approx$ $C$, where $C$ is the best constant in (63).

The validity of inequality (50) with conditions (51) is equivalent to the simultaneous validity of inequalities (53) with (54) and (63) with (64). Therefore, from Propositions 12, 13,14 , and 15 we have the following.

Theorem 16. Let $0 \leq k<n-1$. Let $1<p \leq q<\infty$ and $1<$ $r<\infty$. Suppose that $g$ satisfies conditions (51). Then inequality (50) holds if and only if $D_{k}=\max \left\{D_{k}^{-}, D_{k}^{+}\right\}<\infty$. Moreover, $D_{k} \approx C$, where $C$ is the best constant in (50).

Theorem 17. Let $0 \leq k<n-1$. Let $1<q<p<\infty$ and $1<$ $r<\infty$. Suppose that $g$ satisfies conditions (51). Then inequality (50) holds if $M_{k}=\max \left\{M_{k}^{-}, M_{k}^{+}\right\}<\infty$. Moreover, $M_{k} \approx C$, where $C$ is the best constant in (50).

\section{Competing Interests}

The authors declare that they have no competing interests.

\section{Acknowledgments}

The paper was written under financial support by the Scientific Committee of the Ministry of Education and Science of Kazakhstan, Grant no. 5499/GF4 on priority area "Intellectual Potential of the Country."

\section{References}

[1] R. Oinarov, "Weighted inequalities for one class of integral operators," Doklady Akademii Nauk SSSR, vol. 5, no. 319, pp. 1076-1078, 1991.

[2] S. Bloom and R. Kerman, "Weighted norm inequalities for operators of Hardy type," Proceedings of the American Mathematical Society, vol. 113, no. 1, pp. 135-141, 1991.

[3] T. Chen and G. Sinnamon, "Generalized Hardy operators and normalizing measures," Journal of Inequalities and Applications, vol. 7, no. 6, pp. 829-866, 2002.

[4] D. E. Edmunds and V. D. Stepanov, "On the singular numbers of certain Volterra integral operators," Journal of Functional Analysis, vol. 134, no. 1, pp. 222-246, 1995.

[5] H. P. Heinig and Q. Lai, "Weighted modular inequalities for Hardy-type operators on monotone functions," Journal of
Inequalities in Pure and Applied Mathematics, vol. 1, no. 1, article 10,25 pages, 2000.

[6] V. D. Stepanov, "On the lower bounds for Schatten-vonNeumann norms of certain Volterra integral operators," Journal of the London Mathematical Society, vol. 61, no. 3, pp. 905-922, 2000.

[7] V. D. Stepanov and E. P. Ushakova, "On integral operators with variable limits of integration," Proceedings of the Steklov Institute of Mathematics, vol. 232, pp. 290-309, 2001.

[8] V. D. Stepanov and E. P. Ushakova, "Kernel operators with variable intervals of integration in Lebesgue spaces and applications," Mathematical Inequalities \& Applications, vol. 13, no. 3, pp. 449-510, 2010.

[9] R. Oinarov and A. A. Kalybay, "Three-parameter weighted Hardy type inequalities," Banach Journal of Mathematical Analysis, vol. 2, no. 2, pp. 85-93, 2008.

[10] R. Oinarov and A. A. Kalybay, "Weighted inequalities for a class of semiadditive operators," Annals of Functional Analysis, vol. 6, no. 4, pp. 155-171, 2015.

[11] R. Oinarov, "Two-sided norm estimates for certain classes of integral operators," Proceedings of the Steklov Institute of Mathematics, vol. 204, pp. 205-214, 1994.

[12] A. Kufner, L. Maligranda, and L.-E. Persson, The Hardy Inequality. About Its History and Some Related Results, Vydavatelský Servis, Plzeň, Czech Republic, 2007.

[13] A. Kufner and L.-E. Persson, Weighted Inequalities of Hardy Type, World Scientific Publishing, River Edge, NJ, USA, 2003.

[14] D. Prokhorov and V. Stepanov, "Weighted estimates for a class of sublinear operators," Doklady Mathematics, vol. 88, no. 3, pp. 721-723, 2013.

[15] A. Gogatishvili, R. CH. Mustafayev, and L.-E. Persson, "Some new iterated Hardy-type inequalities," Journal of Function Spaces and Applications, vol. 2012, Article ID 734194, 30 pages, 2012.

[16] A. Gogatishvili, R. Mustafayev, and L.-E. Persson, "Some new iterated Hardy-type inequalities: the case $\theta=1$," Journal of Inequalities and Applications, vol. 2013, article 515, 2013.

[17] D. V. Prokhorov and V. D. Stepanov, "On weighted Hardy inequalities in mixed norms," Proceedings of the Steklov Institute of Mathematics, vol. 283, no. 1, pp. 149-164, 2013. 


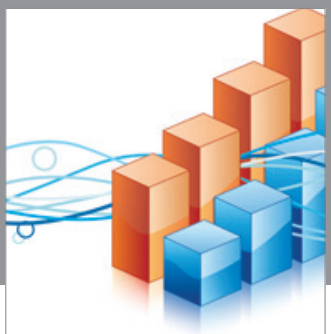

Advances in

Operations Research

vatem alat4

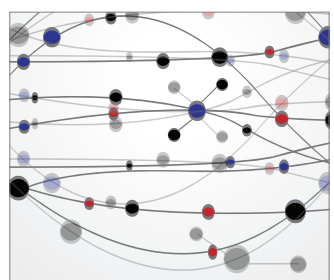

\section{The Scientific} World Journal
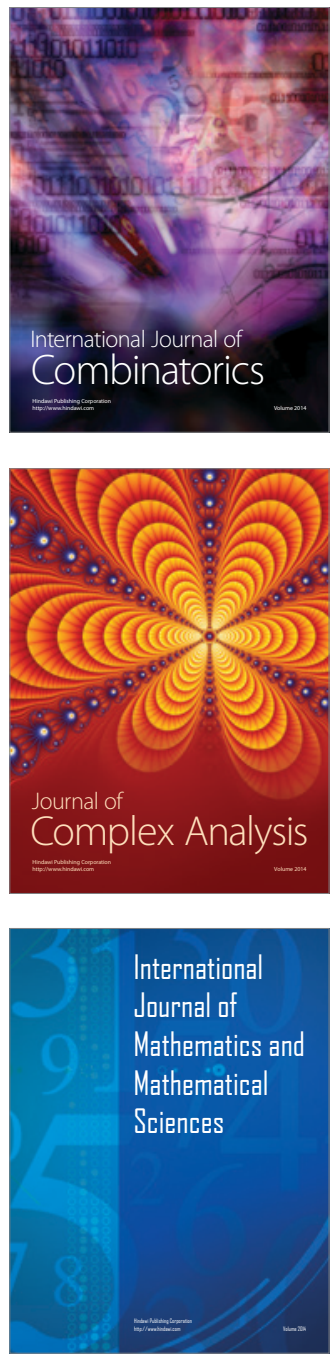
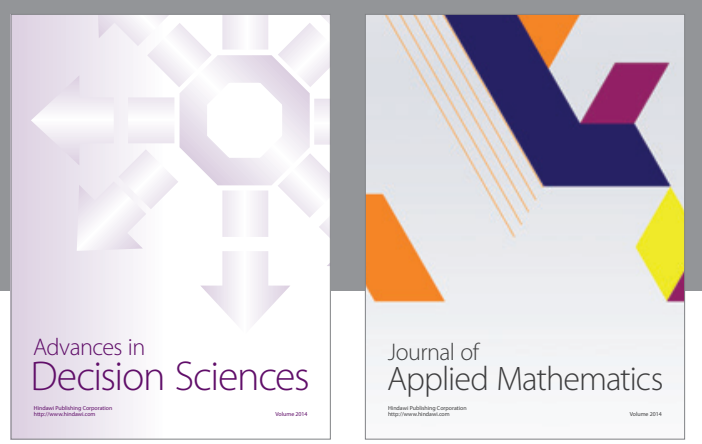

Algebra

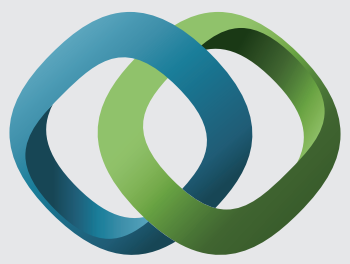

\section{Hindawi}

Submit your manuscripts at

http://www.hindawi.com
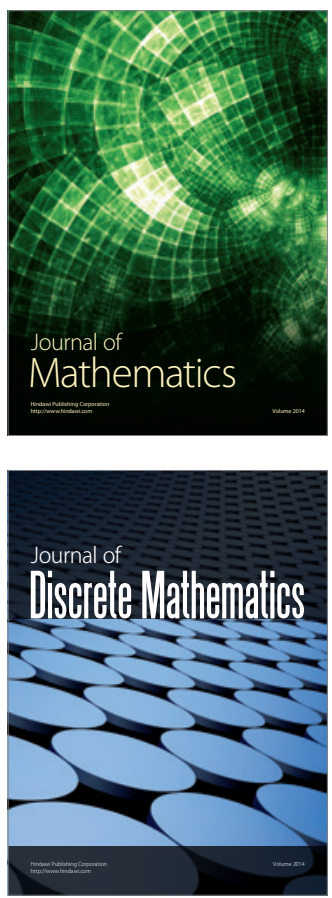

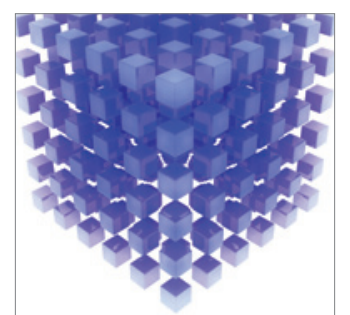

Mathematical Problems in Engineering
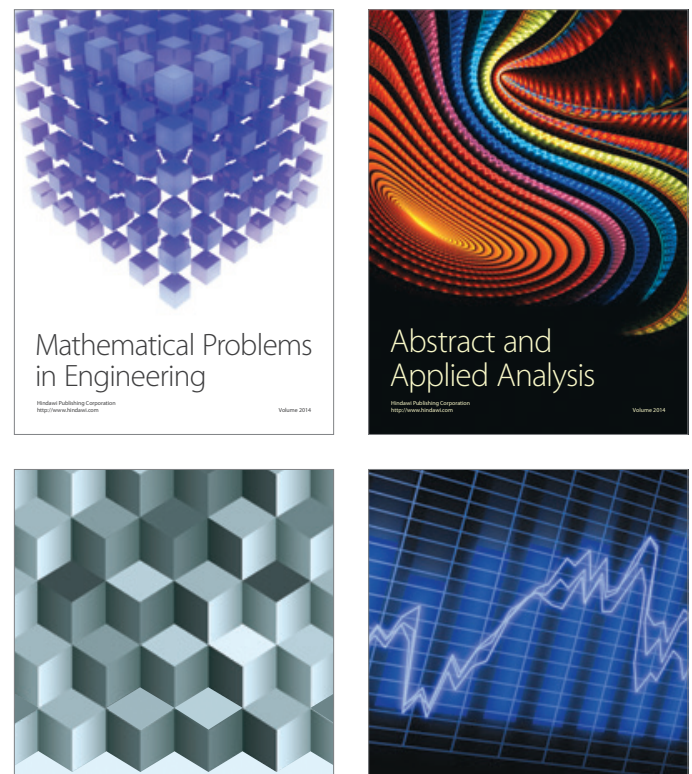

Journal of

Function Spaces

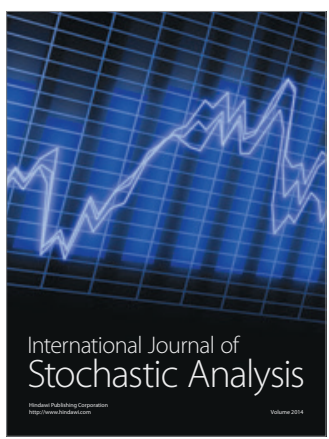

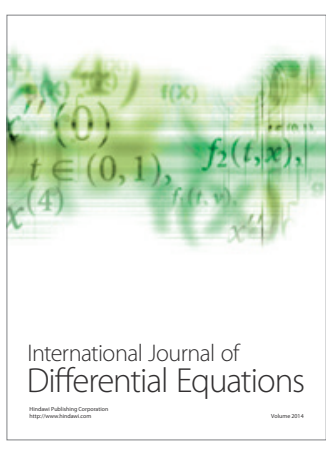
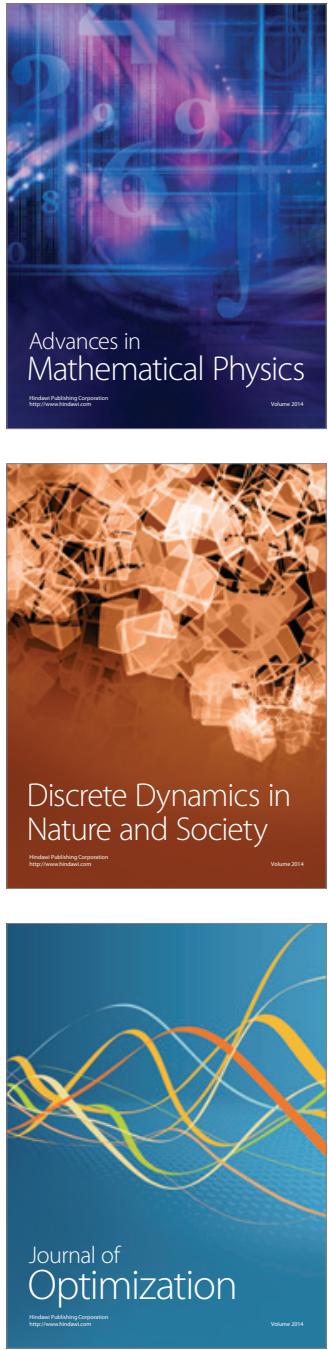